\title{
An Immunohistochemical Study of the Distribution of the Measles Virus Receptors, CD46 and SLAM, in Normal Human Tissues and Subacute Sclerosing Panencephalitis
}

\author{
Stephen McQuaid and Sara Louise Cosby \\ Neuropathology Laboratory (SM), Royal Victoria Hospital, and School of Biology and Biochemistry (SLC), Queen's \\ University Belfast, Belfast, Northern Ireland
}

\begin{abstract}
SUMMARY: We have compared the expression of the known measles virus (MV) receptors, membrane cofactor protein (CD46) and the signaling lymphocyte-activation molecule (SLAM), using immunohistochemistry, in a range of normal peripheral tissues (known to be infected by MV) as well as in normal and subacute sclerosing panencephalitis (SSPE) brain. To increase our understanding of how these receptors could be utilized by wild-type or vaccine strains in vivo, the results have been considered with regard to the known route of infection and systemic spread of MV. Strong staining for CD46 was observed in endothelial cells lining blood vessels and in epithelial cells and tissue macrophages in a wide range of peripheral tissues, as well as in Langerhans' and squamous cells in the skin. In lymphoid tissues and blood, subsets of cells were positive for SLAM, in comparison to CD46, which stained all nucleated cell types. Strong CD46 staining was observed on cerebral endothelium throughout the brain and also on ependymal cells lining the ventricles and choroid plexus. Comparatively weaker CD46 staining was observed on subsets of neurons and oligodendrocytes. In SSPE brain sections, the areas distant from lesion sites and negative for MV by immunocytochemistry showed the same distribution for CD46 as in normal brain. However, cells in lesions, positive for MV, were negative for CD46. Normal brain showed no staining for SLAM, and in SSPE brain only subsets of leukocytes in inflammatory infiltrates were positive. None of the cell types most commonly infected by MV show detectable expression of SLAM, whereas CD46 is much more widely expressed and could fulfill a receptor function for some wild-type strains. In the case of wild-type stains, which are unable to use CD46, a further as yet unknown receptor(s) would be necessary to fully explain the pathology of MV infection. (Lab Invest 2002, 82:403-409).
\end{abstract}

$M$ easles virus (MV) causes approximately one million deaths per year despite the availability of an effective live-attenuated vaccine. Acute infection is associated with initial infection of the respiratory epithelium, with virus spread to lymph nodes followed by viremia and infection of epithelial and endothelial cells (EC) in many peripheral organs, including skin. The latter is responsible for a delayed-type hypersensitivity reaction, giving rise to the typical rash. Complications of MV infection include giant cell pneumonia and otitis media. Infections of the central nervous system (CNS), which are less common, include measles inclusion body encephalitis and subacute sclerosing panencephalitis (SSPE) (Griffin and Bellini, 1996). Neurons and oligodendrocytes are the most common cell types infected in the CNS. However, infection of astrocytes, macrophages, endothelium, and infiltrating lymphocytes has been detected (Allen et al, 1996; Cosby et al, 1989; Kirk et al, 1991).

Received October 15, 2001.

Address reprint requests to: Dr. Stephen McQuaid, Department of Neuropathology, Institute of Pathology, Royal Victoria Hospital, Grosvenor Road, Belfast, Northern Ireland, BT12 6BL. E-mail: s.mcquaid@qub.ac.uk
Membrane cofactor protein (CD46), a regulator of complement-mediated lysis, has been shown to act as a receptor for vaccine strains of MV but not all wild-type strains of the virus (Dorig et al, 1994; Gerlier et al, 1995). CD46 is expressed on all nucleated cells in tissue culture, and in normal tissues CD46 has been found to be widely distributed on lymphocytes, epithelium, and endothelium (Berryman et al, 1993; Franck et al, 1993; Johnstone et al, 1993b; Seya et al, 1988). These are the susceptible cell types for MV in lymphoid tissues and other organs, including skin, kidney, gastrointestinal tract, and liver during childhood measles infection (Moench et al, 1988). We and others have previously established that MV receptor competent CD46 proteins are expressed in brain tissue from patients with persistent CNS MV infections (Buchholz et al, 1996; Ogata et al, 1997), and we have shown that the receptor is absent in MV-positive lesions, suggesting down-regulation of the molecule (Ogata et al, 1997).

More recently the signaling lymphocyte-activation molecule (SLAM) or CDw150, a membrane glycoprotein expressed on primary $B$ cells, activated $T$ cells and memory $\mathrm{T}$ cells, $\mathrm{T}$-cell clones, and immature thymocytes (Sidorenko et al, 1992), has been shown to act as a receptor for both the vaccine strain and 
clinical isolates of MV (Erlenhoefer et al, 2001; Tatsuo et al, 2000). Expression of SLAM has previously been examined in lymphomas (Sidorenko et al, 1992), but expression in peripheral tissues and brain, from normal individuals, has not been investigated. Furthermore, SLAM has not been examined in brain tissue from SSPE patients.

In this study we have compared the expression of both CD46 and SLAM, using immunohistochemistry, in a range of normal peripheral tissues (known to be infected by MV) as well as in normal and SSPE brain. To increase our understanding of how these two receptors could be utilized by wild-type or vaccine strains in vivo, the results have been considered with regard to the known route of infection and systemic spread of MV.

\section{Results}

Preliminary investigations revealed that the $\mathrm{mAb}$ to SLAM was not immunoreactive on formalin-fixed, paraffin-embedded tissue sections despite the use of microwave antigen retrieval. Therefore, cryostat sections were used to assess the distribution of SLAM in tissues. The use of an mAb for detection of SLAM (no polyclonal antibody is available) also did not allow dual labeling with cell-specific mAbs to definitively identify cells types not distinguishable morphologically. Polyclonal antibody to CD46 showed strong immunoreactivity on paraffin sections after microwave antigen retrieval.

\section{Expression of CD46 and SLAM in Peripheral Tissues}

Strong immunohistochemical staining for CD46 was observed in EC lining blood vessels and epithelial cells in a wide range of peripheral tissues including lung alveoli, kidney tubules, skin, lymphoid tissues, and intestine (Fig. 1, a and b). CD46 immunoreactivity was also observed in tissue macrophages, as identified by CD68 positivity on serial sections (Fig. 1a, arrow), and in Langerhans' cells in the dermis layer of the skin, as identified by CD1a positivity on serial sections (Fig. 1, c and d). Very strong CD46 immunoreactivity was also present on the squamous cells in the epidermis of the skin (Fig. 1c). All these cell types showed no expression of SLAM (Fig. 1, e and f). In the germinal center of lymphoid tissues, subsets of cells were positive for SLAM (Fig. 1g), in comparison to CD46, which showed staining of all cell types. In control sections where the primary antibodies were omitted, no staining was observed in any of the tissues.

CD46 labeled all lymphocyte common antigenpositive lymphocytes in peripheral blood preparations (Fig. 1, h and i), whereas only subsets of cells were stained with antibody to SLAM (Fig. 1j). Cell counts on eight high-power fields showed that only $28 \%$ of leukocyte common antigen (LCA)-positive or CD46positive cells were also SLAM positive.

\section{Expression of CD46 and SLAM in Normal and SSPE Brain Tissue}

The distribution of CD46 by cell type and neuroanatomical area in normal brain is shown in Table 1. Strong staining was observed on cerebral EC in all blood vessels throughout the brain (Fig. 2a) and also on ependymal cells lining the ventricles and choroid plexus (Fig. 2b). Comparatively weaker CD46 staining was observed on a subset of neurons (Fig. 2c) and cells identified morphologically as oligodendrocytes in all brain regions examined (Fig. 2a). Small numbers of astrocytes (identified by glial fibrillary acidic protein staining, data not shown) also showed weak staining in the frontal cortex and hippocampus. All cells in the normal brain parenchyma, cerebral EC, and ependymal cells showed no staining for SLAM (Fig. 2, $d$ and e).

In SSPE brain sections, the results for CD46 were as previously described (Ogata et al, 1997). Areas distant from lesion sites and negative for MV by immunocytochemistry showed the same distribution for CD46 as in normal brain (Fig. 2f). However, cells in lesions positive for MV, were negative for CD46, whereas dual-labeling immunocytochemistry revealed MVnegative CD46-positive cells (Fig. 2g).

When cryostat sections of SSPE brain labeled with antibody to SLAM were examined by light and confocal microscopy, all neural cell types (neurons, astrocytes, oligodendrocytes, microglia) and cerebral EC in areas distant from lesions were not stained (Fig. $2 \mathrm{~h}$ ). In MV-positive lesions, with abundant virus present in neurons and oligodendrocytes, SLAM was also not observed in any cells. However, in areas showing perivascuulär inflammation, SLAM-positive leukocytes were present in the infiltrates and in the surrounding brain parenchyma (Fig. 2i).

\section{Discussion}

All isoforms of CD46 have been shown to function as receptors for $\mathrm{MV}$ vaccine strains in tissue culture systems (Bartz et al, 1998; Dorig et al, 1994; Franck et al, 1993), whereas the receptor usage by wild-type strains remains unclear. Bartz et al (1998) have shown that CD46 is only utilized by some wild-type strains and with low binding affinity, whereas Manchester et al (2000) reported that all clinical isolates tested could infect cells in a CD46-dependent manner. In contrast, SLAM has been identified as a receptor for both MV vaccine and wild-type strains (Tatsuo et al, 2000), and it has recently been demonstrated that B-cell lines, which express SLAM and lack a complete CD46, can be used to isolate MV from patients (Ono et al, 2001). To gain an understanding as to how these receptors may be utilized in vivo, it is necessary to determine their expression in normal human tissues and during MV infection.

Many human and monkey cell lines can be infected by MV, whereas MV replication is much more restricted in vivo. Evidence from primate models and human postmortem samples, taken during the early 

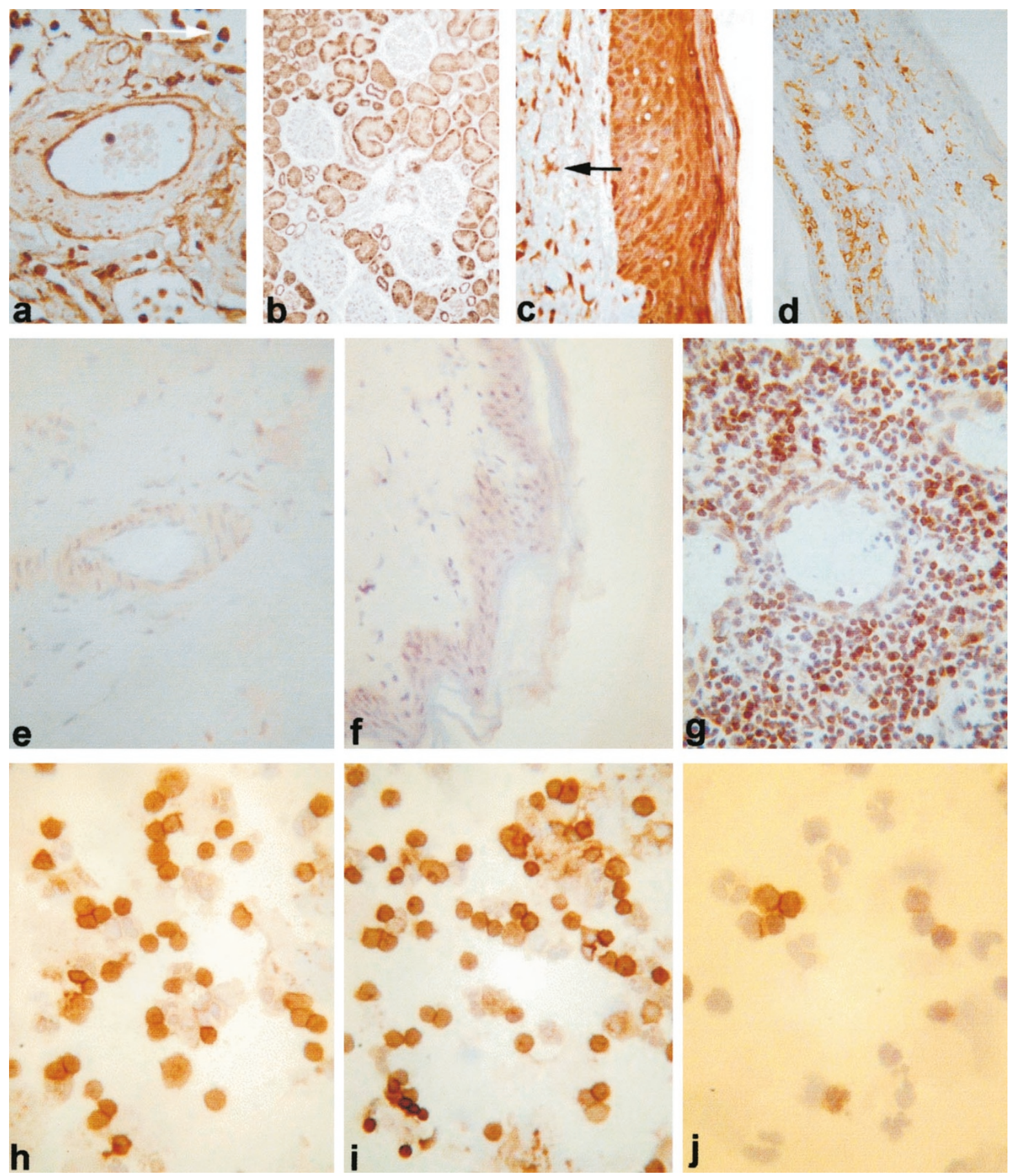

Figure 1.

Membrane cofactor protein (CD46) and signaling lymphocyte-activation molecule (SLAM) immunoreactivity in peripheral tissues. Strong immunoreactivity for CD46 on (a) epithelium and macrophages (arrow) in lung, (b) glomerular epithelium of the kidney, and (c) squamous epithelial cells in the dermis and epidermis of the skin. CD46-positive dendritic cells are also present (arrow) as identified by CD1a positivity on a serial section (d). No expression of SLAM is observed in sections of (e) lung and (f) skin. g, Strong immunoreactivity for SLAM is observed in populations of cells in spleen. $\mathrm{h}$ to $\mathrm{j}$, Cytospin preparations of isolated peripheral blood leukocytes immunostained for (h) LCA, (i) CD46, and (j) SLAM. Note that similar numbers of cells are labeled with antibodies to LCA and CD46, but relatively fewer cells are labeled with mAb to SLAM. All images are digital and were processed in Adobe PhotoShop at $300 \mathrm{dpi}$.

stages of MV infection, shows that the virus enters through the respiratory tract, and initial replication is in tracheal and bronchial epithelial cells (reviewed in Griffin and Bellini, 1996). Although CD46 RNA transcripts are produced in all human tissues by tissuespecific RNA splicing (Johnstone et al, 1993b), it has shown that there is marked variation in expression of CD46 protein between tissues. Strong expression was found on epithelial cells lining exocrine ducts and glands, such as salivary gland and pancreas, and on kidney tubules and glomerular epithelium (Johnstone et al, 1993a). This is in agreement with the results 
Table 1. Distribution of CD46 in Normal Brain as Determined by Immunohistochemistry

\begin{tabular}{lcccc}
\hline & Neurons $(\mathrm{a} / \mathrm{b})$ & ODs $(\mathrm{a} / \mathrm{b})$ & Astrocytes $(\mathrm{a} / \mathrm{b})$ & Endothelium $(\mathrm{a} / \mathrm{b})$ \\
\hline Cerebellum & $+/++$ & $-/-$ & $-/-$ & $++++/++$ \\
Frontal cortex & $+/+$ & $+/+$ & $+/+$ & $++++/++++$ \\
Hippocampus & $+/+$ & $+/+$ & $+/+$ & $++++/++++$ \\
Basal ganglia & $++/++$ & $+/+$ & $+/+$ & $++++/++++$ \\
Corpus callosum & $++/++$ & $+/+$ & $+/+$ & $++++/++++$ \\
Optic nerves & $+/+$ & $+/+$ & $-/-$ & $++++/++++$ \\
Cervical cord & $+/++$ & &
\end{tabular}

ODs, oligodendrocytes.

$\mathrm{a}=$ number of positive cells: $-=$ absent; $+=$ few $;++=$ moderate number; $+++=$ many $;++++=$ abundant .

$\mathrm{b}=$ intensity of immunoreactivity; $-=$ absent; $+=$ weak; $++=$ moderate $;+++=$ intense $++++=$ very intense.

presented in this article, because we found strong expression of CD46 on epithelial cells in a wide range of peripheral tissues, including lung alveoli, kidney tubules, skin, lymphoid tissue, and intestine.

In contrast, the lack of detection of SLAM on epithelial cells is difficult to reconcile with the use of this molecule as a receptor for initial infection of the respiratory tract epithelium. It is possible that inflammatory infiltrates containing SLAM-positive $T$ and $B$ cells, present in the respiratory tract as a result of other unrelated infection or allergens, could allow initial infection. However, for the observed widespread presence of MV antigen in epithelial cells to occur, either another receptor would be necessary or epithelial cells would have to acquire virus by fusing with SLAM-positive inflammatory cells. Fusion of epithelial cells in many tissues is observed during measles infection and is also a characteristic of the MV complication, giant cell pneumonia. Replication of virus in many other tissues, after viremia, occurs in endothelium, giving rise to vasculitis (reviewed in Griffin and Bellini, 1996). Again these cells were heavily stained for CD46 and showed no expression of SLAM. In lymphoid tissue fusion of epithelium also causes the production of Warthin-Finkeldey giant cells (Warthin, 1931). However, there is no evidence to support fusion between either epithelial or endothelial cells and leukocytes.

After initial infection, MV spreads to local lymphatic tissues, with some evidence from monkey studies that suggests that this may occur via pulmonary macrophages (Kamahora and Nii, 1961; Sergiev et al, 1960). We have shown that tissue macrophages, including those in the lung, are positive for CD46 but fail to stain for SLAM. It has been previously reported (Cocks et al, 1995; Sidorenko et al, 1992) that CD14 ${ }^{+}$monocytes are negative for SLAM, whereas a recent study (Ohgimoto et al, 2001) has found that dendritic cells (DC), derived from these, express SLAM. We have shown that SLAM does not seem to be induced to any significant level after differentiation of monocytes to macrophages, which indicates that different lineages of cells, derived from monocytes, vary in expression of SLAM.

Studies in primate models have shown that after spread to the lymphatic system, MV replication occurs in thymus, spleen, and lymph nodes as well as appendix and tonsils (reviewed in Griffin and Bellini, 1996).
Virus RNA has been demonstrated in esterasepositive cells in the blood of infected children and in postmortem spleen tissue, indicating that monocyte/ macrophages rather than lymphocytes are primarily infected (Brown et al, 1989). In agreement with previous studies (Johnstone et al, 1993a), we have shown that all nucleated cells in blood as well as all cells in the germinal centers of the spleen and lymph nodes show expression of CD46. In contrast, SLAM staining was only observed on a subset of cells in both blood and the germinal centers of lymphoid tissues. Along with $\mathrm{B}$ and $\mathrm{T}$ cells, CD34 ${ }^{+}$bone marrow-derived DC are also present in lymphoid and other tissues, including skin (Banchereau and Steinman, 1998), and have been shown to be infected by MV in vitro (Fugier-Vivier et al, 1997; Grosjean et al, 1997; Schnorr et al, 1997; Steineur et al, 1998). In this study, staining of the resident population of skin DC, Langerhans cells, proved positive for CD46 but negative for SLAM, indicating that the latter is unlikely to act as a receptor for infection of bone-derived DC.

The route of MV infection of the CNS is unknown but may occur either by direct infection of EC or by passage of infected leukocytes across the blood-brain barrier as demonstrated in canine distemper infection in dogs (Appel, 1969). We have previously reported infection of cerebral EC in cases of SSPE (Kirk et al, 1991), and in this study we show that as for peripheral EC, these cells express a high level of CD46 but not SLAM. All cell types in normal brain sections were negative for SLAM, and only a subset of cells in the inflammatory infiltrate were SLAM positive in SSPE brain tissue. We have previously shown that leukocytes in perivascular cuffs in SSPE contain MV RNA and antigen (Allen et al, 1996; Cosby et al, 1989). It is possible that virus could be carried into the CNS in infected leukocytes or infection of these cells could occur after initial infection of EC, as previously suggested (Kirk et al, 1991).

In SSPE, neurons and oligodendrocytes are the most commonly infected cells with occasional astrocyte infection (Allen et al, 1996). None of these cells showed staining for SLAM, and only a small proportion of neurons and oligodendrocytes showed relatively weak staining for CD46. This indicates that a further receptor(s) would be required by all strains of $\mathrm{MV}$ to directly infect neural cells at a level observed in 

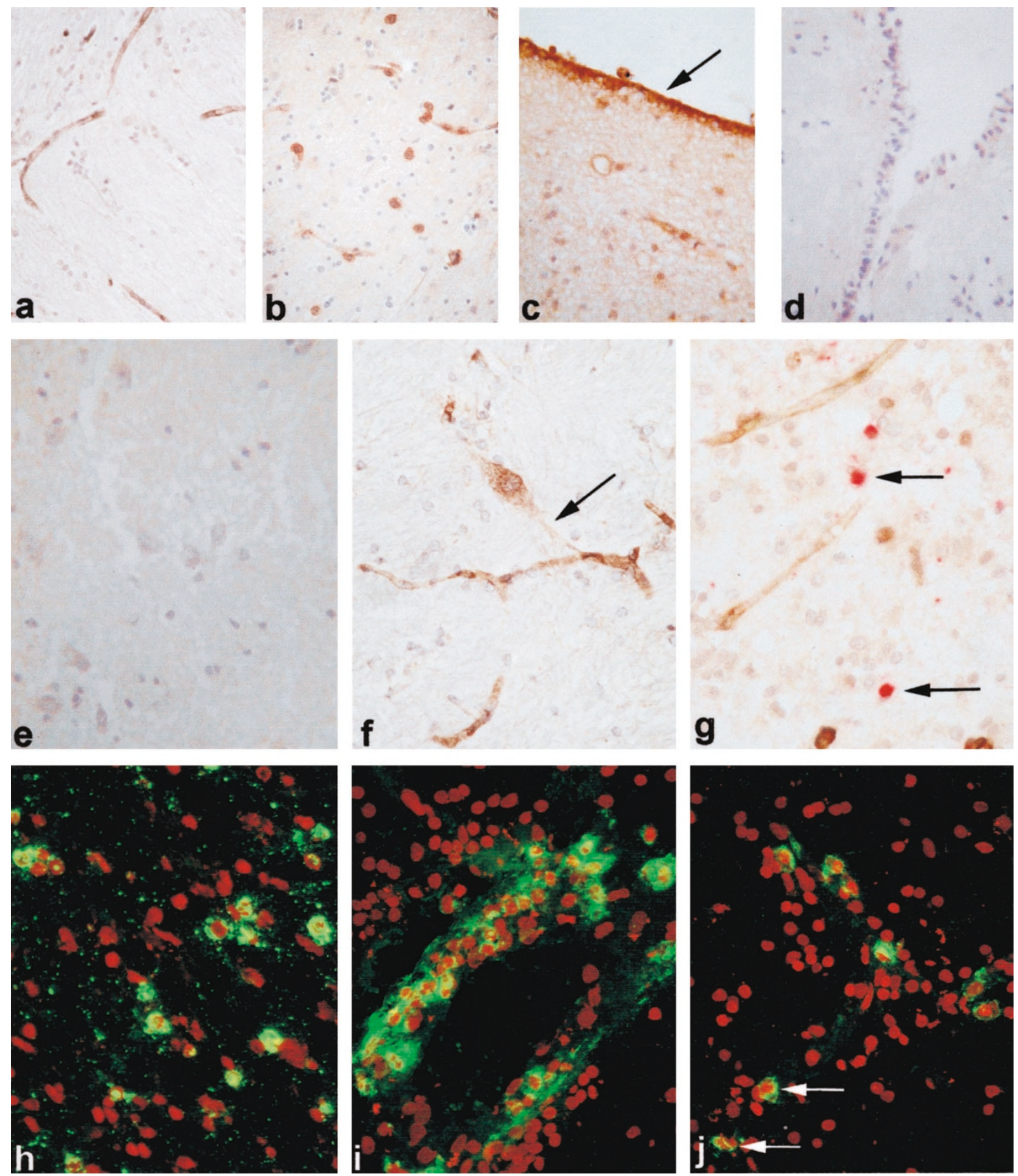

\section{Figure 2.}

CD46 and SLAM immunoreactivity in brain tissue. Strong immunoreactivity for CD46 in normal brain on (a) endothelium in the cortex, (b) endothelium and oligodendrocytes in the hippocampus, and (c) ependymal cells lining the ventricle (arrow). However, in normal brain SLAM is not expressed on (d) ependymal cells or (e) cells in the brain parenchyma. In measles virus (MV)-positive subacute sclerosing panencephalitis (SSPE) brain sections, CD46 expression is observed on (f) endothelium and the cell body and processes of neurons (arrow). g, Double labeling of CD46 (diaminobenzidine-brown) and MV-positive cells (arrowed, red), demonstrates no dual labeling of individual cells. $\mathrm{h}$ to j, Frozen section of SSPE brain tissue (h) immunostained for MV. Note MV-positive neurons and presence of virus in cell processes. i, Strong expression of SLAM on cells within a perivascular cuff. j, A small number of SLAM-positive cells are present in a cuff and also infiltrating into the surrounding brain parenchyma (arrows). All images are digital and were processed in Adobe PhotoShop at $300 \mathrm{dpi}$.

SSPE tissue. Alternatively, wild-type strains, which could use CD46 as a receptor, could infect a percentage of cells directly, including a limited number of neurons, and spread throughout the CNS transsynaptically (Allen et al, 1996; Lawrence et al, 2000). We have previously shown that MV-infected cells in SSPE are negative for CD46 and visa versa (Ogata et al, 1997), suggesting that virus may down-regulate this molecule and could therefore use it as a receptor in the CNS. However, although both of these functions 
require interaction of the virus hemagglutinin protein with CD46, they can be independent (Bartz et al, 1998; Galbraith et al, 1998).

A further possibility is spread of virus from infected ECs to neurons via cell-to-cell processes. This is further supported by a previous in vitro study in which we demonstrated that spread of virus from neuroepithelial to neuronal cells could occur along cell processes (McQuaid et al, 1998). It is also possible that MV could enter the brain via the choroid plexus, as occurs with mumps virus (Takano et al, 1999). Once again our results indicate that either CD46 or a receptor(s) other than SLAM would have to be utilized.

In conclusion, the evidence from our own and other laboratories indicates that measles primarily infects epithelial cells, ECs, and monocytes/macrophages in the periphery, while neurons and oligodendrocytes are preferentially infected in the CNS. We have found that none of these cells show detectable expression of SLAM, although it is possible that very low levels could be present and allow some degree of infection. CD46 is much more widely expressed and could fulfill a receptor function for vaccine strains in vivo and in vitro as well as possibly some wild-type strains. However, in the case of wild-type strains that are unable to use CD46 (Bartz et al, 1998; Ono et al, 2001), a further as yet unknown receptor(s) would be necessary to fully explain the pathology of MV infection.

\section{Materials and Methods}

\section{Tissues}

Peripheral and CNS tissues were obtained from a patient who died with no apparent signs of neurologic disease. Four autopsy cases of SSPE were included on the basis of clinical features, cerebrospinal fluid, and serum measles IgG antibody titers and pathologic examination (Allen et al, 1996). Tissue from all cases was fixed in $10 \%$ formalin for 2 weeks before processing to paraffin. Peripheral and brain tissue blocks from the normal case and brain blocks from the SSPE cases were also snap-frozen in liquid nitrogen-cooled isopentane. Cytospin preparations of peripheral blood leukocytes from a normal individual were prepared as previously described (Brankin et al, 1996).

\section{Antibodies and Immunocytochemistry}

A polyclonal antibody that recognizes all isoforms of CD46 (1:200) was a gift from F. Wild (Institut Pasteur de Lyon, France). mAbs were also obtained to SLAM (1:1000; Kamiya Biomedical Company, Thousand Oaks, California); to MV nucleocapsid protein (1:2000; Harlan Sera-Lab, Crawley Down, Sussex, United Kingdom); to leukocyte common antigen (LCA) (1:50; DAKO, Bucks, United Kingdom); and to CD1a (1:20; Novocastra Labs, Newcastle, United Kingdom).

Cryostat sections $(12 \mu \mathrm{m})$ were cut from snap-frozen tissues and fixed in 10\% formalin. After blocking endogenous peroxidase in $0.5 \% \mathrm{H}_{2} \mathrm{O}_{2}$ in methanol for 10 minutes, sections were incubated in polyclonal anti-
CD46 or mAbs to SLAM or MV nucleocapsid overnight at $4^{\circ} \mathrm{C}$. For paraffin sections a microwave antigen retrieval step was used for CD46, MV, LCA, and CD1a as described previously (McQuaid et al, 1995). For both cryostat and paraffin sections, immunoperoxidasebound antibodies were detected as described previously using diaminobenzidine as peroxidase substrate (Allen et al, 1996). Sections were counterstained with hematoxylin, and assessment and photomicroscopy were performed on a Leitz Aristoplan microscope.

Alternatively, bound antibodies were detected by immunofluorescence using Alexa 488 rabbit anti-mouse (monoclonal) or Alexa 488 goat anti-rabbit (polyclonal) secondary antibodies. Nuclei were then counterstained in propidium iodide ( $2 \mu \mathrm{g} / \mathrm{ml}$; Sigma Chemical, Poole, Dorset, United Kingdom) for 30 seconds and washed in PBS, and the sections were mounted using Citiflour (Amersham Corporation, Paisley, United Kingdom). A Leica TCS/NT confocal scanning laser microscope equipped with a krypton/argon laser was used to examine the samples for fluorescence. Alexa 488-labeled samples were visualized by excitation at $488 \mathrm{~nm}$ with a 506-538 band-pass emission filter.

In dual-labeling experiments on paraffin sections from SSPE brain tissue, CD46 was applied first and reaction sites were visualized with peroxidasediaminobenzidine. Subsequently antibodies to MV, glial fibrillary acidic protein (1:100; Dako) for astrocyte or CD68 (1:50, Dako) for macrophage identification, were applied and detected using streptavidin-alkaline phosphatase and Vector Red substrate.

\section{References}

Allen IV, McQuaid S, McMahon J, Kirk J, and McConnell R (1996). The significance of measles virus antigen and genome distribution in the CNS in SSPE for mechanisms of viral spread and demyelination. J Neuropathol Exp Neurol 55:471-480.

Appel MJ (1969). Pathogenesis of canine distemper. Am J Vet Res 30:1167-1182.

Banchereau J and Steinman RM (1998). Dendritic cells and the control of immunity. Nature 392:245-252.

Bartz R, Firsching R, Rima B, ter Meulen V, and SchneiderSchaulies J (1998). Differential receptor usage by measles virus strains. J Gen Virol 79:1015-1025.

Berryman M, Franck Z, and Bretscher A (1993). Ezrin is concentrated in the apical microvilli of a wide variety of epithelial cells whereas moesin is found primarily in endothelial cells. J Cell Sci 105:1025-1043.

Brankin B, Osman M, Herlihy L, Hawkins SA, and Cosby SL (1996). Failure to detect measles virus RNA, by reverse transcriptionpolymerase chain reaction, in peripheral blood leukocytes of patients with multiple sclerosis. Mult Scler 1:204-206.

Brown HR, Goller NL, Rudelli RD, Dymecki J, and Wisniewski HM (1989). Postmortem detection of measles virus in nonneural tissues in subacute sclerosing panencephalitis. Ann Neurol 26:263-268.

Buchholz CJ, Gerlier D, Hu A, Cathomen T, Liszewski MK, Atkinson JP, and Cattaneo R (1996). Selective expression of a subset of measles virus receptor-competent CD46 isoforms in human brain. Virology 217:349-355. 
Cocks BG, Chang CC, Carballido JM, Yssel H, de Vries JE, and Aversa G (1995). A novel receptor involved in T-cell activation. Nature 376:260-263.

Cosby SL, McQuaid S, Taylor MJ, Bailey M, Rima BK, Martin SJ, and Allen IV (1989). Examination of eight cases of multiple sclerosis and 56 neurological and non-neurological controls for genomic sequences of measles virus, canine distemper virus, simian virus 5 and rubella virus. J Gen Virol 70:2027-2036.

Dorig RE, Marcil A, and Richardson CD (1994). CD46, a primate-specific receptor for measles virus. Trends Microbiol 2:312-318.

Erlenhoefer C, Wurzer WJ, Loffler S, Schneider-Schaulies S, ter Meulen V, and Schneider-Schaulies J (2001). CD150 (SLAM) is a receptor for measles virus but is not involved in viral contact-mediated proliferation inhibition. J Virol 75: 4499-4505.

Franck Z, Gary R, and Bretscher A (1993). Moesin, like ezrin, colocalizes with actin in the cortical cytoskeleton in cultured cells, but its expression is more variable. J Cell Sci 105:219231.

Fugier-Vivier I, Servet-Delprat C, Rivailler P, Rissoan MC, Liu YJ, and Rabourdin-Combe C (1997). Measles virus suppresses cell-mediated immunity by interfering with the survival and functions of dendritic and T cells. J Exp Med 186:813-823

Galbraith SE, Tiwari A, Baron MD, Lund BT, Barrett T, and Cosby SL (1998). Morbillivirus downregulation of CD46. J Virol 72:10292-10297.

Gerlier D, Varior-Krishnan G, and Devaux P (1995). CD46mediated measles virus entry: A first key to host-range specificity. Trends Microbiol 3:338-345.

Griffin D and Bellini W (1996). Measles virus. In: Field BN, Knipe DM, Howley PM, Chanock RM, Melnick, JL, Monath TP, Roizman B, and Straus SE, editors. Fields virology. Philidelphia-New York: Lippincott-Raven, 1267-1312.

Grosjean I, Caux C, Bella C, Berger I, Wild F, Banchereau J, and Kaiserlian D (1997). Measles virus infects human dendritic cells and blocks their allostimulatory properties for CD4 ${ }^{+}$T cells. J Exp Med 186:801-812.

Johnstone RW, Loveland BE, and McKenzie IFC (1993a). Identification and quantification of complement regulator CD46 on normal human tissues. Immunology 79:341-347.

Johnstone RW, Russell SM, Loveland BE, and McKenzie IF (1993b). Polymorphic expression of CD46 protein isoforms due to tissue-specific RNA splicing. Mol Immunol 30:1231-1241.

Kamahora J and Nii S (1961). Pathological and immunological studies of monkeys infected with measles virus. Arch Ges Virusforch 16:161-167.

Kirk J, Zhou A-L, McQuaid S, Cosby SL, and Allen IV (1991). Cerebral endothelial cell infection by measles virus in subacute sclerosing panencephalitis: Ultrastructural and in situ hybridization evidence. Neuropathol Appl Neurobiol 17:289-297.

Lawrence DM, Patterson CE, Gales TL, D'Orazio JL, Vaughn MM, and Rall GF (2000). Measles virus spread between neurons requires cell contact but not CD46 expression, syncytium formation, or extracellular virus production. J Virol 74:1908-1918.

Manchester M, Eto DS, Valsamakis A, Liton PB, FernandezMunoz R, Rota PA, Bellini WJ, Forthal DN, and Oldstone MB (2000). Clinical isolates of measles virus use CD46 as a cellular receptor. J Virol 74:3967-3974.
McQuaid S, Campbell S, Wallace IJ, Kirk J, and Cosby SL (1998). Measles virus infection and replication in undifferentiated and differentiated human neuronal cells in culture. J Virol 72:5245-5250.

McQuaid S, McConnell R, McMahon J, and Herron B (1995). Microwave antigen retrieval for immunocytochemistry on formalin-fixed paraffin-embedded postmortem CNS tissue. J Pathol 176:207-216.

Moench TR, Griffin DE, Obriecht CR, Vaisberg AJ, and Johnson RT (1988). Acute measles in patients with and without neurological involvement: Distribution of measles virus antigen and RNA. J Infect Dis 158:433-442.

Ogata A, Czub S, Ogata S, Cosby SL, McQuaid S, Budka H, ter Meulen V, and Schneider-Schaulies J (1997). Absence of measles virus receptor (CD46) in lesions of subacute sclerosing panencephalitis (SSPE) brains. Acta Neuropathologica 94:444-449.

Ohgimoto S, Ohgimoto K, Niewiesk S, Klagge IM, Pfeuffer J, Johnston IC, Schneider-Schaulies J, Weidmann A, ter Meulen V, and Schneider-Schaulies S (2001). The haemagglutinin protein is an important determinant of measles virus tropism for dendritic cells in vitro. J Gen Virol 82:1835-1844.

Ono N, Tatsuo H, Hidaka Y, Aoki T, Minagawa H, and Yanagi $Y$ (2001). Measles viruses on throat swabs from measles patients use signaling lymphocytic activation molecule (CDw150) but not CD46 as a cellular receptor. J Virol 75: 4399-4401.

Schnorr JJ, Seufert M, Schlender J, Borst J, Johnston IC, ter Meulen V, and Schneider-Schaulies S (1997). Cell cycle arrest rather than apoptosis is associated with measles virus contact-mediated immunosuppression in vitro. J Gen Virol 78:3217-3226.

Sergiev PG, Ryzantseva NE, and Shroid IG (1960). The dynamics of pathological processes in experimental measles in monkeys. Acta Virol (Eng) 4:265-273.

Seya T, Ballard AL, Bora NS, Kumar V, Cui W, and Atkinson JP (1988). Distribution of membrane cofactor protein (MCP) of complement on human peripheral blood cells. Eur J Immunol 18:1289-1294.

Sidorenko SP, Vetrova EP, Yurchenko OV, Berdova AG, Shlapatskaya LN, and Gluzman DF (1992). Monoclonal antibodies of IPO series against B cell differentiation antigens in leukemia and lymphoma immunophenotyping. Neoplasma 39:3-9.

Steineur MP, Grosjean I, Bella C, and Kaiserlian D (1998). Langerhans cells are susceptible to measles virus infection and actively suppress $\mathrm{T}$ cell proliferation. Eur $\mathrm{J}$ Dermatol 8:413-420.

Takano T, Takikita S, and Shimada M (1999). Experimental mumps virus-induced hydrocephalus: viral neurotropism and neuronal maturity. Neuroreport 10:2215-2221.

Tatsuo H, Ono N, Tanaka K, and Yanagi Y (2000). SLAM (CDw150) is a cellular receptor for measles virus. Nature 406:893-897.

Warthin AS (1931). Occurrence of numerous large giant cells in the tonsils and pharyngeal mucosa in the prodromal stage of measles. Arch Pathol 11:864-874. 